\title{
PRESUPUESTOS DESDE EL DERECHO COMPARADO PARA EL PERFECCIONAMIENTO DE LA REGULACIÓN LEGAL DE LA ASIMETRÍA MUNICIPAL EN CUBA
}

PRINCIPLES FROM COMPARATIVE LAW FOR THE IMPROVEMENT OF THE LEGAL REGULATION OF MUNICIPAL ASYMMETRY IN CUBA ORÇAMENTOS DO DIREITO COMPARADO PARA A MELHORIA DA REGULAMENTAÇÃO LEGAL DA ASSIMETRIA MUNICIPAL EM CUBA

\section{DRA. C. ORISEL HERNÁNDEZ AGUILAR ${ }^{1}$ LIC. MÓNICA ÁLVAREZ CHÁVEZ²}

RESUMEN: A pesar de que en Cuba el municipio es responsable de satisfacer las necesidades mínimas locales, este se encuentra enmarcado en una regulación que homogeniza sus principales aspectos, lo que provoca que una merma en su funcionalidad que no le permite un desempeño acorde a la asimetría de las realidades locales. Para dar tratamiento a esta problemática el presente trabajo ofrece, con base en análisis históricos, doctrinales y comparados, los presupuestos teórico-jurídicos que deben guiar el perfeccionamiento de la regulación legal de la asimetría municipal en Cuba.

PALABRAS CLAVES: municipio, asimetría municipal, regulación legal

ABSTRACT: While in Cuba, the municipalities are responsible for the local minimum needs, they are framed in a regulation that homogenize their main aspects, which causes a decline in its functionality that does not allow a performance according to the asymmetry of the local realities. To give treatment to this problem this paperwork was proposed, with base in the historical, doctrinal and comparative analysis, the theoretical-legal presupposition that should guide the improvement of the legal regulation of the heterogeneity of municipalities in Cuba.

KEY WORDS: municipality, municipal asymmetry, legal regulation

RESUMO: Embora em Cuba o município seja responsável por satisfazer as necessidades mínimas locais, esta é enquadrada em um regulamento que homogeneiza seus principais aspectos, o que provoca uma diminuição de sua funcionalidade que não permite uma performance de acordo com a assimetria de as realidades locais. Para dar tratamento a este problema, o presente trabalho oferece, com base em análises históricas, doutrinais e comparativas, os pressupostos teórico-jurídicos que devem orientar a melhoria da regulamentação legal da assimetria municipal em Cuba.

PALAVRAS-CHAVE: município, assimetria municipal, regulamentação legal

1 Doctora en Ciencias Jurídicas. Profesora de Derecho de la Universidad de Pinar del Río, Cuba. oriselha@upr.edu.cu

2 Licenciada en Derecho. Consultora jurídica de la Empresa Provincial de Servicios Legales de Pinar del Río. monica.fidel@nauta.cu 


\section{INTRODUCCIÓN}

Una de las cuestiones, que ha estado presente desde el surgimiento de las estructuras municipales - constituyendo a su vez una de sus problemáticas- ha sido el hecho de que cada una de ellas representa realidades diferentes. La causa de esta heterogeneidad se ha debido a la existencia de un mapa municipal, en cada país, conformado por entes de varios tamaños cuya configuración no es uniforme. Las diversidades manifestadas entre ellos se deben a factores como: su extensión superficial, organización institucional, alcance competencial de sus órganos, recursos naturales, humanos y económicos lo que conduce a la necesidad de la existencia de regulaciones específicas que se atemperen a cada una de estas realidades disímiles entre sí.

En Cuba la Carta Magna expresa que el municipio constituye una "sociedad local con capacidad para satisfacer las necesidades mínimas locales" (Constitución de la República de Cuba, 2002, Art. 102). Un análisis de este recepto permite comprender que, para cumplir el cometido asignado, se deben tomar en cuenta las particularidades propias de las comunidades. No obstante, la regulación legal vigente no asume la existencia de la asimetría municipal, lo que propicia que no sea la idónea la regulación de las aristas organizativo - funcionales de los órganos locales para enfrentar los problemas existentes en cada localidad.

Todo ello ha conllevado a que los municipios cubanos no estén competencialmente preparados para satisfacer a cabalidad las necesidades de la comunidad; que se recienta la eficacia de la división política administrativa en que están insertados; que no aprovechen al máximo los recursos naturales de que disponen; que no cuenten con presupuestos propios y adecuados para financiarse; que no instrumenten de forma efectiva políticas públicas y, por ende, no puedan convertirse en los gestores idóneos del desarrollo local, entre otras consecuencias. Lo que se traduce, al decir de la profesora Prieto Valdés (2015, p. 156), como se expresaba anteriormente, en una merma de su funcionalidad que no les permite desplegar el máximo de sus potencialidades.

Es precisamente por ello que, en el actual contexto cubano que apuesta por el perfeccionamiento de la institucionalidad ( $C f r$. Lineamientos de la Política Económica y Social del Partido y la Revolución, 2012), resulta pertinente hablar de todos los aspectos de la realidad que pueden y deben ser cambiados. 
Ante tal premisa, este trabajo se propone fundamentar los presupuestos teóricojurídicos que deben guiar el perfeccionamiento de la regulación legal de la asimetría municipal en Cuba.

Para ello se parte de sistematizar el tratamiento doctrinal que ha recibido la asimetría municipal y analizar el tratamiento legal que brindan los ordenamientos jurídicos de Hispanoamérica a la misma. Sobre la base de los resultados de estas tareas se determinan cuales se pueden considerar los antecedentes del tratamiento de la asimetría municipal en Cuba, para finalmente argumentar los presupuestos teórico-jurídicos que deben sustentar su adecuada regulación en la Isla.

\section{CONSIDERACIONES DOCTRINALES SOBRE LA ASIMETRÍA MUNICIPAL}

En opinión de Carmona Romay (1962, p. 58) el municipio, en razón a su gobierno y administración, constituye la organización político-administrativa de la sociedad local. Siguiendo con este enfoque, debe admitirse que la estructura sociogeográfica de la comunidad se caracteriza por ser compleja, dinámica -interna y externamente-, y creciente. De tal manera que se puede afirmar, que las localidades son diversas entre sí, porque cada una de ellas representan una realidad diferente y ostentan necesidades propias. Lo antes dicho constituye la manifestación fáctica de la existencia de una marcada asimetría entre las municipalidades, la que requiere, a su vez, un pronunciamiento del ordenamiento jurídico que esté encaminado a reconocer lo que objetivamente ocurre, de modo que se le dé un tratamiento adecuado.

A la existencia de un mapa municipal de diferentes dimensiones se le denomina "asimetría". Su reconocimiento -y ahí radica su importancia-sienta las pautas para fundamentar que la organización institucional local no tiene que ser idéntica para todas las unidades, que el rango competencial de los diferentes entes municipales puede ser diferente, y que junto al régimen jurídico general pueden cohabitar tratamientos especiales o diferenciados para determinados municipios (Villabella Armengol,2006, p. 158). 
Cuando se analizan las razones que justifican su existencia, se adoptan distintos criterios que ponderan aristas muy diversas y que competen a áreas del conocimiento dispares, como la geografía, la economía, la sociología, entre otras. Para ello, se toman como referencia aspectos muy puntuales y variados como la concentración de la población, su composición etaria, sus necesidades, sus índices de consumo, así como la extensión del territorio, sus límites geográficos, los recursos naturales que en él se encuentran, la historia de las comunidades, las particularidades medioambientales y otros factores que debido a su carácter variable devienen en causas de la aludida disparidad entre localidades.

Al respecto se pronuncian algunos autores (Bullón Ramírez, 1959, pp. 5-10; Morell Ocaña, 1972, pp. 35-53 y Jiménez Morales, 2009, p. 3) en obras diversas que, aunque no todas se enfocan hacia una comprensión cabal de la asimetría, sí ofrecen evidencia de la existencia de la misma. Entre ellos vale la pena destacar el criterio sostenido por la profesora Prieto Valdés (2015, p. 156), quien ofrece una sistematización bastante amplia de los elementos teóricos sobre los que existe un mayor pronunciamiento en la doctrina, cuando apunta que la extensión territorial, la composición poblacional, sociocultural y cuantitativa, los recursos naturales, el desarrollo económico, los intereses prevalecientes, los grupos políticos que intervienen en la toma de decisiones y las tradiciones, son elementos que marcan la heterogeneidad existente entre las municipalidades, y las dotan de sus propias particularidades.

Según se constata del análisis antes referido, los elementos mencionados se pueden comprender dentro de dos grandes criterios: el de población y el de territorio. Así pues, la población puede ser enfocada desde la composición etaria, la concentración demográfica, la movilidad de la misma según sus necesidades, los índices de consumo, el nivel cultural adquirido, la historia del grupo humano, el crecimiento o decrecimiento tanto por movimiento natural (nacimientos y defunciones), como por emigración o inmigración, entre otros. Lo que explica que este criterio tenga múltiples manifestaciones. Del otro lado, al abordar el territorio se pueden tratar aspectos como, la extensión, los límites geográficos, los recursos naturales, las características del suelo, la ubicación en áreas urbanas o rurales y el clima, entre otros.

De lo expuesto se colige que, dada la capacidad de comprender multiplicidad de cuestiones, los criterios población y territorio constituyen los elementos básicos a considerar para regular apropiadamente la asimetría, siempre que se tome en cuenta que cada uno de 
estos, en su interior, cuenta con una composición variada de aristas que evidencian la disparidad que se manifiesta entre las localidades. Lo anterior no desdice que se puedan asumir criterios más particularizados, que se enfoquen en aspectos puntuales de las realidades antes descritas. No obstante, a los efectos de esta investigación, se propone tomar como referencia los aludidos criterios con el carácter de básicos para enfocar la mencionada nota, dado su carácter sistematizador.

\section{LA REGULACIÓN DE LA ASIMETRÍA MUNICIPAL EN LOS ORDENAMIENTOS JURÍDICOS DE HISPANOAMÉRICA}

En la práctica analizar el desarrollo de las instituciones jurídicas de otros países resulta siempre un referente de gran validez. El presente estudio focaliza su atención en el área hispanoamericana, por cuanto ofrece la posibilidad de operar dentro de ordenamientos jurídicos cercanos a la tradición y cultura jurídicas cubanas, con los que se comparte el mismo sistema de Derecho. Para ello se centrará la atención en los textos constitucionales y en las leyes ordinarias que competen al ámbito local, con el propósito de evidenciar los niveles de reconocimiento de la asimetría municipal en estas normas.

\subsection{LA REGULACIÓN DE LA ASIMETRÍA MUNICIPAL EN LOS TEXTOS CONSTITUCIONALES DE} HISPANOAMÉRICA

La asimetría se prevé en doce (12) de las Cartas Magnas analizadas, lo que representa aproximadamente un sesenta y tres por ciento $(63 \%)$ del total que la regulan.

En su tratamiento prevalecen de forma general como criterios el de población, referido a los pueblos originarios (este criterio representa el treinta y cuatro por ciento (34\%) del total de veces que se menciona la asimetría) en sus distintas tipologías, el de territorio asociado a la situación geográfica (este criterio representa el siete por ciento (7\%) del total de veces que se menciona la asimetría), la importancia económica (este criterio representa el diez por ciento $(10 \%)$ del total de veces que se menciona la asimetría), la ecología (rste criterio representa el siete por ciento $(7 \%)$ del total de veces que se menciona la asimetría), entre otros. 
Su presencia no siempre se aprecia de una forma clara, ni a través de elementos concretos, ya que existen normas en las que no se establece un criterio, pero sí se evidencia un mandato al legislador ordinario para que se reconozca la misma, constituyendo un ejemplo de ello Argentina (Constitución Política de la República de Argentina, 1994, Art. 5).

En este estudio se constató que desde los años noventa hasta la actualidad es una constante su previsión en las Constituciones del área. Dentro de los textos estudiados vale destacar los de Colombia (Constitución Política de la República de Colombia, 1991, Arts. 7, 286, 289 y 320) y Paraguay (Constitución de la República del Paraguay, 1992, Arts. 140, 159 y 171) que se reconocen varios criterios para la determinación de la asimetría, como el territorio (situación y ubicación geográfica, importancia económica, ecología) y la población (cantidad de habitantes).

\subsection{LA REGULACIÓN DE LA ASIMETRÍA EN LAS LEYES MUNICIPALES DE HISPANOAMÉRICA}

En las leyes municipales, de acuerdo a su propia naturaleza de constituir normas de inferior jerarquía y su obligación de regular con mayor precisión las relaciones municipales, se aprecia un tratamiento más acabado de la asimetría municipal. Su presencia se evidencia en doce (12) países, cifra que representa el sesenta y tres por ciento (63\%) del total.

En estas normas los criterios que más se aprecian son la población según la cantidad de habitantes (este criterio representa el veinte por ciento $(20 \%)$ del total de veces que se menciona la asimetría), y la población tomando en cuanto a cultura e historia (este criterio representa el ocho por ciento ( $8 \%$ ) del total de veces que se menciona la asimetría), tomados en consideración para la creación de municipios. Además, se introducen nuevos aspectos que particularizan a determinados comunidades como, el territorio referido a las actividades turísticas, mineras, industriales, las zonas de mejoramiento residencial (Vid. Ley $\mathrm{N}^{\circ}$ 81, Ley de Municipios Autónomos del Estado Libre Asociado de Puerto Rico, 1991, Art. 16.004), entre otros, y la importancia estratégica de determinadas sociedades locales, así como su interés nacional.

El tracto histórico de esta nota en las leyes municipales se comporta, también, de la misma forma que en los textos constitucionales, manteniéndose uniformidad en los años 90 en 
la regulación del elemento población, referido a los pueblos de acuerdo a su origen. Por otra parte, se observa cierto crecimiento de criterios hacia el dos mil en lo adelante.

\section{LA REGULACIÓN LEGAL DE LA ASIMETRÍA MUNICIPAL EN CUBA}

Durante las diferentes etapas por las que ha atravesado la historia cubana, han estado vigentes disímiles cuerpos legales que se han ocupado de la materia municipal. A continuación, se realiza un análisis de estas normas, con el fin de conocer si en ellas se evidencian pronunciamientos en pos de reconocer la asimetría municipal.

Las Ordenanzas de Cáceres, redactadas en el año 1573, reconocían las disparidades que existían entre las municipalidades. Así, se aprecia como el redactor de estas normativas tuvo en cuenta las particularidades de determinadas localidades. Como expresara el profesor Carreras Collado, Cáceres al preparar las ordenanzas tomó en consideración las características de La Habana, de acuerdo a su condición de principal centro comercial de Cuba y, al mismo tiempo, atendió las dificultades de las demás villas, en especial las de Bayamo que, por su riqueza, situación geográfica, sus feraces tierras, su población, y el espíritu batallador de sus vecinos, reclamaba una atención muy especial (Carreras Collado, 1981, pp. 40 y 41).

Este cuerpo legal estuvo vigente hasta que, por Real Decreto de fecha de 27 de julio de 1857, fue sustituido por las Ordenanzas de Concha, a partir de las cuales se estableció un nuevo régimen municipal que abogaba por la uniformidad, y rompía con la lógica de las libertades y de la descentralización imperante hasta ese momento (Vid. Carrera Jústiz, 1905, pp. 299-301).

Luego de la firma del Pacto del Zanjón se promulgó en la Isla la Ley Municipal de 1878. En esta ley se aprecia que la asimetría subyace en la base de las normas pues el legislador en ocasiones distingue los municipios unos de otros, en razón a la cantidad de habitantes, con el fin de establecer los regímenes de multas y la cuantía de votos necesarios para formar acuerdo (Ley Municipal de la Península, aplicada para la isla de Cuba, 1878, Arts. 77,98 у 149).

De todo lo antes dicho se colige que los actos normativos ocupados de las cuestiones referentes a los municipios, que rigieron durante la etapa colonial en Cuba, reconocieron la 
asimetría adoptando como criterios para ello el de población asociado a la cantidad de habitantes, de forma preponderante, $y$, en menor medida, algunas características puntuales de los territorios.

Una vez que Cuba dejó de ser colonia de España cambió su condición a neocolonia de la gran potencia americana, produciéndose a partir de ese momento un importante período de auge de las regulaciones locales municipales. En este contexto el primer cuerpo legal en promulgarse fue la Constitución de 1901. En ella la asimetría no se tuvo en cuenta, lo que terminó desencadenando en el "establecimiento de una sola forma de gobierno municipal, la que el constituyente de entonces creyó mejor, incurriendo en un absolutismo doctrinal, que conducía al sometimiento de cada municipalidad a un solo sistema" (Angulo y Pérez, 1941, p. 11).

Esta situación subsistió hasta la puesta en vigor de la Ley Orgánica de los Municipios en el año 1908. Esta norma consagró la asimetría a través de la clasificación de los municipios en tres clases de acuerdo al criterio población, en cuanto al número de residentes en cada localidad (Decreto N. ${ }^{\circ}$ 568. Ley Orgánica de los Municipios, 1908, Arts. 9 y 55).

Otro momento importante, de la historia municipal cubana, fue la promulgación de la Constitución de 1940, la que retomó muchos de los contenidos desarrollados en las normativas anteriores, plasmándolos de forma más acabada y haciéndose eco de las doctrinas municipales del momento epocal.

La Carta Magna del 40 llevó a su más alta expresión la asimetría al admitir que esta se reflejara mediante la posibilidad de los municipios de adoptar un sistema de gobierno entre varios, eligiendo aquel que fuese más adecuado a su realidad, y el derecho de darse su propia Carta Orgánica (Constitución de la República de Cuba, 1940, Art. 222).

En el período revolucionario no se ofrecen referencias jurídicas al respecto. En las décadas del 70 y el 80 se puso el énfasis en procurar la uniformidad y el rápido desarrollo de los municipios desde una política centralista.

La Constitución de la República correspondiente al año 1976, que consagra en su parte dogmática las grandes conquistas sociales, políticas y económicas logradas durante los años del poder revolucionario, y en su parte orgánica al nuevo aparato del poder, conocido como el Sistema de Órganos del Poder Popular, instaura un modelo, aún vigente, que se caracteriza por su carácter generalmente uniformador. Aunque dentro de él se han admitido algunas 
manifestaciones, mayormente organizativas de diversidad, están lejos de configurar una previsión de la asimetría.

\section{PRESUPUESTOS TEÓRICO-JURÍDICOS PARA UNA ADECUADA REGULACIÓN LEGAL DE LA ASIMETRÍA MUNICIPAL EN CUBA}

En base a lo que se ha expresado anteriormente, y tomando en cuenta el estado del debate, en la doctrina y en el derecho comparado al respecto, queda puesto de manifiesto que en nuestro país no se aprecia un reconocimiento adecuado de la asimetría municipal, en virtud de las realidades imperantes. Se hace por ello pertinente plantear los presupuestos esenciales para perfeccionar su regulación, a fin de que esta se corresponda con la realidad material de las municipalidades patrias y viabilice la funcionalidad de las estructuras existentes, para dar cumplimiento así a mandato constitucional de satisfacer las necesidades mínimas locales.

\subsection{RECONOCIMIENTO CON RANGO CONSTITUCIONAL DE LA ASIMETRÍA MUNICIPAL}

La norma constitucional se ubica en la base y cima del ordenamiento jurídico de cualquier país. Al decir de Pérez Royo (1997, p. 309) "lo específico de la Constitución es que con ella solo cabe la perspectiva general, es solo una y su objeto es la construcción jurídica del orden político global de la sociedad". La misma solo refrenda los postulados fundamentales de cada país, los que constituyen, a su vez, referentes para el resto de las leyes que a ella se subordinan, cuyos espíritus nunca deben contradecirla.

Un contenido esencialmente constitucional es el que se refiere a la organización de los poderes públicos, al cual se dedica por excelencia la parte orgánica de la Ley Suprema, y dentro de ella la normación de la institución local reviste gran relevancia, puesto que los Estados tienen en la misma su cédula fundamental, es decir su eslabón básico. De ahí que, combinando la importancia que ostentan los dictados constitucionales, por su lugar dentro del ordenamiento jurídico, y el hecho de que es insoslayable la presencia de un pronunciamiento de ese carácter general sobre la mencionada institución local, sea importante que esa 
regulación se ocupe de tratar los elementos esenciales que deben regir a esta última, figurando dentro de ellos la asimetría municipal.

En el caso cubano, la Carta Magna establece que los municipios constituyen sociedades locales, organizadas políticamente, con capacidad para satisfacer las necesidades mínimas locales, lo que supone que el éxito de su gestión debe pasar por atender en su funcionamiento, las particularidades propias de sus comunidades. Sin embargo, la norma de referencia no sienta los preceptos necesarios para reconocer, y tratar jurídicamente las disparidades existentes dentro del mapa municipal patrio, por lo que se requiere un pronunciamiento en pos de ello, a fin de dar una mejor coherencia a los pilares sociopolíticos e ideológicos que animan al Estado y que la propia lex superior consagra (Matilla Correa, 2015, p. 190).

Para técnicamente hacer que se consagre el mencionado principio, la norma constitucional debe cumplir con los requerimientos de mantener "un predominio de enunciados de principio o enunciados valorativos" (Bono López, 2000, p. 178), que sienten las pautas para que en otras normas de inferior rango se desarrollen dichos postulados, enriqueciendo el discurso. En razón de ello se necesita que la redacción de sus preceptos no sea excesivamente pormenorizada, larga o explicativa, y que obedezca a una lógica sistémica, de forma tal que se pueda obtener un orden legal marcado por la coherencia. A lo anterior se suma la necesidad de que exista una exposición ordenada de sus contenidos.

Como se expuso supra, lo primero que se percibe del estudio del derecho comparado es que en la mayoría de los textos constitucionales hispanoamericanos más modernos hay presencia de la asimetría. Por ello, teniendo en cuenta, además, lo mejor de la tradición municipal cubana y en consonancia con lo hasta aquí expuesto en materia de técnica, se puede sostener que el tratamiento constitucional más completo de este elemento, sería aquel que reconozca mediante un precepto la existencia de un mapa municipal heterogéneo en el contexto nacional cubano y deje sentadas las pautas para su tratamiento posterior en una ley de inferior jerarquía.

Ello podría hacerse tomando como paradigma el caso ecuatoriano, de forma tal que se dejaran sentados de partida los presupuestos básicos que deben ser apreciados a la hora de regular esta realidad (Constitución Política de la República del Ecuador, 2008, Art. 242). Los mismos variarán según los intereses del legislador nacional, pudiendo figurar como criterios 
que denotan asimetría, por ejemplo, las particularidades propias de los territorios como sus recursos económicos o medioambientales, las características socio-demográficas de la población, entre otros.

En consecuencia, la Carta Magna cubana, respetando la técnica jurídica, teniendo en cuenta la tradición municipal histórica, el estado del derecho comparado, la realidad fáctica de diferenciación de las municipalidades patrias, debe regular la asimetría con rango constitucional. Siempre sobre la base de un carácter lo suficientemente abstracto, pero completo que deje sentadas las pautas a fin de asegurar el desarrollo posterior de su alcance y contenido en la correspondiente ley municipal.

\subsection{Promulgación de unA Ley Municipal QUe CONSAGRE A LA ASIMETRÍA}

\section{A) Una Ley Municipal para Cuba.}

Como es lógico, de la sistemática jurídica derivada de un texto constitucional deben crearse un cúmulo de leyes, que desarrollen las instituciones básicas para el correcto desenvolvimiento de la sociedad y del Estado. Entre las que, indispensablemente, requieren la existencia de una norma que las organicen figuran las municipalidades.

El primer problema que se encuentra al respecto, en el ordenamiento jurídico cubano, es la clara ausencia de un sistema de fuentes (Matilla Correa, 2006, pp. 38-39). Esto nos enfrenta a la inexistencia de un catálogo ordenado, por gradación, de los cuerpos normativos, que se pueden desarrollar en el país, que sirva de referencia, además, para saber el contenido que deben de tener cada una de estas normas, toda vez que los cuerpos legales ostentan jerarquía por su rango y contenido.

A pesar de esta realidad imperante, en el contexto patrio se asume que el tipo de acto normativo que sigue en jerarquía a la Carta Magna son las leyes, emitidas por la Asamblea Nacional del Poder Popular (Constitución de la República de Cuba, 2002, Art. 70) -órgano legislativo-, pues a ellas les corresponde desarrollar, de preferencia, los contenidos constitucionales que son básicos para la subsistencia del sistema, dentro de los que se encuentra la organización y el funcionamiento municipal. En tal sentido se puede aludir que, la ley "es el instrumento normativo principal en rango del sistema de Derecho nacional, y a 
ella se subordina el resto de la preceptiva que crean los entes que tienen las potestas normandi" (Matilla Correa, 2006, p. 62).

Por ello ante la carencia real y la falta sistemática en cuanto a la institución municipal, reconocida en Cuba, existe un pronunciamiento mayoritario en pos de que se cree una Ley Municipal.

Para regular en la Isla la institución del municipio, no se puede soslayar el reconocimiento de la asimetría municipal que termina traduciéndose en la funcionalidad del mismo, por eso dada la significación de sus contenidos se requieren que sean ordenados en el mencionado cuerpo legal. En el contexto cubano entre los argumentos que abalan esta propuesta se encuentran en: las necesidades de perfeccionamiento de la institucionalidad (Pérez Hernández, y Prieto Valdés, 2004, pp. 187-191); de integrar la legislación dispersa sobre el tema (Pérez Hernández y Díaz Legón, 2015, p. 383-385); así como ser consecuente con la tradición histórica local, que contó en su momento con una Ley Municipal sobre esta materia que regulaba, a su vez, el referido principio.

También varios académicos y operadores que se desempeñan dentro de la esfera municipal abogan, en el actual contexto cubano, por la existencia de una ley con estas características, como se puede constatar de la lectura de la obra colectiva, “¿Qué municipio queremos? Respuestas para Cuba en clave de descentralización y desarrollo local”. En tal sentido, los primeros, como resultado de diferentes investigaciones, coinciden en cuanto a la importancia de crear esta nueva norma, con el fin de ordenar las instituciones que son básicas para el buen funcionamiento de los municipios.

Estas propuestas de los académicos están en consonancia con los resultados de las encuestas practicadas a los cuadros en las provincias de Artemisa y Mayabeque, en las cuales se identificaron como debilidades, en el actual desarrollo municipal, la carencia de una ley municipal, así como también la negación práctica de la heterogeneidad como principio de constitución y funcionamiento municipal, resultado que se arrojó mediante el proyecto investigativo: "La descentralización municipal en pos del desarrollo local y la actualización del modelo económico” (Pérez Hernández y Díaz Legón, 2015, p. 190).

En consecuencia, la futura Ley de los municipios cubanos una vez que establezca qué entiende por municipio, y partiendo de un enunciado constitucional, como se viene proponiendo en este trabajo, que siente las bases al respecto, debe regular la asimetría 
municipal -que constituye un pilar básico de la institución local, y por ende es merecedor de este rango-, teniendo en cuenta las pautas que se proponen a continuación.

\section{B) Reconocimiento de la asimetría por medio de una clasificación de municipios}

La asimetría, como ya se había explicado en esta investigación, constituye una traducción a la estipulación en ley de la realidad objetivamente diferenciada de las municipalidades que integran un país.

En el caso cubano esta realidad material ha sido tratada por los Congresos del Partido Comunista de Cuba y por los estudios realizados en los últimos años, no solo desde la ciencia del Derecho sino también a partir de la Geografía. Desde esta ciencia se han desarrollado estudios, enfocados en problemáticas distintas, que coinciden en apuntar a la ausencia de uniformidad en los territorios como elementos a considerar para propiciar un adecuado tratamiento de las más disímiles problemáticas de que se ocupan (Cfr. Font Aranda, 2001; Martínez Mesa, 2011 y Iñiguez Rojas, 2014).

En tal sentido, lo que se espera de una futura Ley Municipal es que haga un reconocimiento de esa situación fáctica en la norma. El mencionado pronunciamiento podría estar encaminado hacia la realización de una clasificación de los municipios. Un primer criterio a tener en cuenta sería el de la cantidad de habitantes, posibilidad que no resulta extraña a la historia patria, pues en el año 1908, a tenor de la Ley Orgánica de los Municipios, se estableció una clasificación en cuanto este criterio, además de que en la actualidad constituye una regularidad en el derecho comparado como se vio anteriormente en esta investigación. El otro criterio a considerar para distinguir las clases de municipios podría ser la ubicación geográfica de los territorios, nótese en este sentido que se ha sugerido la posibilidad del reconocimiento de municipios metropolitanos (Pérez Hernández y Díaz Legón, 2015, p.386), en razón de las particularidades y complejidades de determinadas localidades.

También podría establecerse una diferenciación de municipalidades en cuanto a la actividad económica, criterio seguido por países como España y Venezuela. Esto resultaría pertinente en la Isla debido al interés que despiertan determinadas actividades, por las que se apuesta para el progreso futuro de la economía nacional: la zona especial del Mariel y el 
desarrollo del turismo. Con respecto a este último particular se podrían catalogar determinadas localidades como municipios con vocación turística, según lo establece el profesor Menoya Zayas (2015, p. 21) cuando expresa, que los mismos presentan rasgos diferenciales con respecto a un municipio común o con otro tipo de vocación, y en este sentido se enfrentan a problemáticas adicionales relativas a la prestación de servicios, tanto turísticos como ordinarios. Constituyen un lugar atractivo para potenciales visitantes de acuerdo a sus especiales condiciones naturales y culturales, por lo que requieren una regulación especial adecuada a las realidades que enfrenta.

Por otro lado, y tomando como referencia la ley puertorriqueña, valdría la pena considerarla posibilidad de designar municipios como zonas de mejoramiento residencial. Estos, de acuerdo a las particularidades que posee nuestro país, pueden ser instrumentados legalmente con el fin de brindarles una atención diferenciada a todas aquellas localidades que presentan situaciones de desgaste desde el punto de vista constructivo, empobrecimiento, malas condiciones de higiene, etc. Especialmente la provincia de La Habana podría resultar beneficiada con este particular pues, como se expresa en la segunda parte de la obra "Las tantas Habanas: estrategia para comprender sus dinámicas sociales”, se constata que en ella hay una especial proclividad a la presencia de problemas y conflictos sociales en aquellos entornos marcados por el deterioro de viviendas y los derrumbes, lo que hace que requiera un especial tratamiento por parte de las autoridades.

\section{CONCLUSIONES}

El reconocimiento legal de la asimetría resulta una exigencia básica para atemperar las estructuras y funciones de los entes locales a las realidades concretas de sus comunidades. En tal sentido las normas hispanoamericanas han asumido, progresivamente, varios criterios para la determinación de la asimetría partiendo de las peculiaridades de sus territorios y población.

La legislación vigente en Cuba carece de una previsión ordenadora de la asimetría. Por tal razón, ante la política actual de perfeccionamiento institucional, resulta oportuno considerar la introducción en la Carta Magna cubana de un pronunciamiento en esta dirección 
que siente las pautas para que una futura Ley Municipal pueda establecer una clasificación de los municipios. Para realizar esta catalogación pueden seguirse los criterios de población y ubicación geográfica, distinguirse determinados municipios por la importancia de la actividad económica que en ellos se desarrolla, y dársele especial tratamiento a los que se encuentran en zonas que requieren mejoramiento residencial.

\section{REFERENCIAS BIBLIOGRÁFICAS}

ANGUlO Y PÉREZ, Andrés. La Afirmación y la Quiebra de la Libertad de las Instituciones Locales. La Habana: s/e, 1941, 17 p.

BONO LÓPEZ, María. La racionalidad lingüística en la producción legislativa. CARBONELl, Miguel; PEDROZA DE LA LLAVE, Susana Thalía (Coordinadores). Elementos de técnica legislativa. México: UNAM, 2000, pp. 159-194.

BULLÓN RAMÍREZ, Antonio. La población municipal. Evolución histórica de su clasificación y efectos de la misma en la legislación vigente. Madrid: Instituto de Estudios de la Administración Local, 1959, 201 p.

CARMONA ROMAY, Adriano G.: Ofensa y defensa de la Escuela Sociológica del municipio. Revista de la Facultad de Derecho de la Universidad de Zulia, Maracaibo, n. 4, enero-abril 1962, pp. 47-59.

CARRERAS COLLADO, Julio. A.: Historia del Estado y del Derecho en Cuba, La Habana: Editorial Pueblo y Educación, 1981, 559 p.

CARRERA JÚSTIZ, Francisco. Introducción a la Historia de las Instituciones locales de Cuba, Tomo II, La Habana: Lib. é Imp. La Moderna Poesía, 1905, 510 p.

FERNÁNDEZ BUlté, Julio. Historia del Estado y el Derecho en Cuba, La Habana: Editorial Félix Varela, 2005, 256 p.

FONT ARANDA, Mabel. Estudio geográfico ambiental del municipio y la ciudad de Santa Clara como base para el perfeccionamiento del sistema de vigilancia en salud. Tesis presentada en opción al grado científico de Doctora en Ciencias Geográficas. Facultad de Geografía, Universidad de La Habana. La Habana: s/e, 2001, 80 p.

IÑIGUEZ ROJAS, Luisa (Coordinadora): Las tantas habanas: estrategia para comprender sus dinámicas sociales. La Habana: Editorial UH, 2014, 406 p. 
JIMÉNEZ MORALES, Caridad Rosa. El Distrito en el Estado Cubano: Premisas para su ordenación jurídica. Tesis presentada en opción al grado académico de Doctora en Ciencias Jurídicas, Universidad de Oriente. Santiago de Cuba: s/e, 2009, 132 p.

MARTÍNEZ MESA, Midelkys. Diagnóstico ambiental de los Barrios y los Focos Insalubres del municipio Cerro. Trabajo de Curso, Facultad de Geografía, Universidad de La Habana. La Habana: s/e, 2011, 83 p.

MATILLA CORREA, Andry. Comentarios sobre las Fuentes del Derecho Administrativo Cubano. COLECTIVO DE AUTORES. Temas de Derecho Administrativo, Tomo I, La Habana: Editorial Félix Varela, 2006, pp. 33-219.

MATILLA CORREA, Andry. Municipio y administración local: breves glosas al hilo del contexto jurídico cubano actual. PÉREZ HERNÁNDEZ, Lissette; DÍAZ LEGÓN, Orestes. ¿Qué municipio queremos? Respuestas para Cuba en clave de descentralización y desarrollo local. La Habana: Editorial UH, 2015, pp. 175-192.

MENOYA ZAYAS, Sandys. Modelo de gestión del turismo desde el gobierno local para municipios con vocación turística basado en el enfoque de cadena de valor, Tesis presentada en opción al grado académico de Doctor en Ciencias Económicas, Universidad de Pinar del Río. Pinar del Río: s/e, 2015, 118 p.

MORELL OCAÑA, Luis. Estructuras locales y ordenación del espacio, Madrid: Instituto de Estudios de Administración Local, 1972, 251 p.

PÉREZ HERNÁNDEZ, Lissette; PRIETO VALDÉS, Martha. Estado, Gobierno y administración. Diferenciación conceptual a propósito de las Ley de los Municipios. PÉREZ HERNÁNDEZ, Lissette; PRIETO VALDÉS, Martha. Temas de Derecho Constitucional Cubano, La Habana: Félix Varela, pp. 187-191.

PÉREZ ROYO, Javier. Curso de derecho constitucional, Segunda Edición. Madrid: Marcial Pons, 1997, $805 \mathrm{p}$.

POSADA, Adolfo. Escritos municipalitas y de la vida local. Madrid: Instituto de Estudios de Administración Local, 1979, 489 p.

PRIETO VALDÉS, Martha. ¿Qué municipio necesitamos? Algunos principios para su configuración. PÉREZ HERNÁNDEZ, Lissette; DÍAZ LEGÓN, Orestes (Coordinadores). ¿Qué municipio queremos? Respuestas para Cuba en clave de descentralización y desarrollo local. La Habana: Editorial UH, 2015, pp.155-174.

VI CONGRESO DEL PARTIDO COMUNISTA DE CUBA. Lineamientos de la Política Económica y Social del Partido y la Revolución. Disponible online en http://www.prensalatina.cu/Dossiers/LineamientosVICongresoPCC.pdf, consultado el 1/07/2012.

VILLABELLA ARMENGOL, Carlos. Los principios teóricos del municipio. Su apreciación en la municipalidad cubana. PÉREZ HERNÁNDEZ, Lissette; PRIETO VALDÉS, Martha (Compiladoras). Temas de Derecho Constitucional Cubano. La Habana: Editorial Félix Varela, 2006, pp. 158-173. 


\section{LEGISLACIÓN}

ARGEnTINA. Constitución Política de la República de Argentina, Santa Fe, 22/8/1994. Disponible en http://www.boletin oficial.gob.ar/datos/cons_es.pdf Consultado el 7 de mayo de 2017.

COLOMBIA. Constitución Política de la República de Colombia, Gaceta Constitucional N ${ }^{0}$ 114 y No 116 , de 4/07/1991 y 20/07/1991.

CUBA. Constitución de la República de Cuba, Gaceta Oficial de la República de Cuba N ${ }^{\circ}$ 464, La Habana, 8/07/1940.

CUBA. Constitución de la República de Cuba (vigente), Gaceta Oficial de la República de Cuba, Edición Especial, La Habana, 24/02/1976, reformada por la Ley de Reforma Constitucional, Gaceta Oficial de la República de Cuba, Edición Extraordinaria, No 9, La Habana, 13/07/1992 y por la Ley de Reforma Constitucional, Gaceta Oficial de la República de Cuba, Edición Extraordinaria, No 10, La Habana, 16/07/2002.

CUBA. Decreto N. ${ }^{\circ}$ 568. Ley Orgánica de los Municipios, Gaceta Oficial de la República de Cuba, edición extraordinaria, La Habana, 29 de mayo de 1908.

ECUADOR. Constitución Política de la República del Ecuador, Registro Oficial, Quito, 20/10/2008.

ESPAÑA. Ley Municipal de la Península, aplicada para la isla de Cuba. Gaceta de Madrid, No. 204 y No. 251 de 25 de julio de 1878 y 28 de julio de 1878. Gaceta, colección histórica: ayuda y contenido. Agencia Estatal. Boletín Oficial del Estado Español. Disponible en www.boe.es Consultado el 21 de octubre de 2011.

PARAGUAY. Constitución de la República del Paraguay, Asunción, 20/06/1992. Disponible en http://www.gacetaoficial.gov.py/datos/cons_es.pdf Consultado el 25 de septiembre de 2016.

PUERTO RICO. Ley N ${ }^{\circ}$ 81, Ley de Municipios Autónomos del Estado Libre Asociado de Puerto Rico de 30/08/1991. Disponible en http://www.gobierno.pr/NR/rdonlyres/ 77F59993-5F78-401F-B234-4F577FB24C4D/0/Ley81Revisada.pdf Consultado el 21 de enero de 2014. 